\title{
ONE-SIDED CLUSTER-SET THEORY IN A POLYDISC
}

\author{
C. L. CHILDRESS AND G. L. CSORDAS
}

\begin{abstract}
In this paper, we develop a technique which reduces the investigation of the boundary behavior of a single function $f$ in $H^{\infty}\left(U^{n}\right), n>1$, to the study of the cluster sets of a sequence of functions in $H^{\infty}(U)$. We also demonstrate the significance and usefulness of one-sided cluster-set theory.
\end{abstract}

1. Introduction. The purpose of this paper is threefold. First, we develop a technique which reduces the investigation of the boundary behavior of a single function $f$ in $H^{\infty}\left(U^{n}\right), n>1$, to the study of the cluster sets of a sequence of functions in $H^{\infty}(U)$. This technique was inspired to a large extent by the ingenious methods and ideas which were first introduced in the one-variable theory of cluster sets by Doob [5], [6]. (In particular, see Doob [5, p. 441, Theorem 4.2].) At the outset, we impose a limitation on the possible approaches to a boundary point $P$ on $T^{n}$. Thus, at least initially, we restrict ourselves to only one-sided cluster sets. (For the definition, see \$2.) One advantage of this restricted approach to a boundary point $P$ on $T^{n}$ is that several fundamental results of the one-variable theory of cluster sets become tractable in the several-variable situation. For example, in Theorem 3 we are able to extend the one-sided Iversen theorem to the polydisc $U^{n}$. In addition to the one-sided approach, another ingredient of our technique is contained in the statements and in the proofs of Lemmas 4 and 5. Second, we use our technique to prove a strong form of the Gross Cluster-Value Theorem [8] in the polydisc $U^{n}$ (Theorem 6 ). Third, we provide some applications of our results. For instance, in Corollary 9 we extend and generalize Lindelöf's important classical theorem on the asymptotic values of analytic functions [10]. We end the paper with an open question concerning the GehringLohwater phenomenon [7] in the polydisc $U^{n}$.

For the sake of simplicity, we present our theorems in the unit polydisc $U^{2}$. We remark, however, that our results remain valid for a polydisc in $\mathbf{C}^{n}$, where $n \geqslant 2$.

The authors wish to thank Professor A. J. Lohwater for the many fruitful conversations which they have had with him.

Received by the editors October 12, 1978.

AMS (MOS) subject classifications (1970). Primary 30A72.

Key words and phrases. One-sided cluster sets, angular and principal cluster sets, the IversenDoob Theorem, the Lindelö Theorem, the Gross Cluster-Value Theorem, the Gehring-Lohwater Theorem. 
2. Definitions and terminology. The topological concepts we use in this paper are relative to the extended complex plane $\mathbf{C}_{\infty}$. We denote the complement of a set $A$ by $\tilde{A}$.

Let the function $\zeta=f(z, w)$ map $U^{2}$ into $\mathbf{C}_{\infty}$. Let $\gamma_{1}$ and $\gamma_{2}$ be two paths in $U$ which terminate at $e^{i \theta_{1}}$ and $e^{i \theta_{2}}$, respectively. (We use the terms "path" and "arc" interchangeably.) If $\gamma=\gamma_{1} \times \gamma_{2}$, then we call the cluster set $C_{\gamma}\left(f,\left(e^{i \theta_{1}}, e^{i \theta_{2}}\right)\right)$ the product path cluster set of $f$ at $\left(e^{i \theta_{1}}, e^{i \theta_{2}}\right)$. This cluster set is defined as the set of all values $\zeta \in \mathbf{C}_{\infty}$ such that there is a sequence $\left\{\left(z_{n}, w_{n}\right)\right\}$ with the properties that $\left(z_{n}, w_{n}\right) \in \gamma$ for each $n,\left(z_{n}, w_{n}\right) \rightarrow\left(e^{i \theta_{1}}, e^{i \theta_{2}}\right)$, and $f\left(z_{n}, w_{n}\right) \rightarrow \zeta$. If both $\gamma_{1}$ and $\gamma_{2}$ lie in a Stolz angle, then we call $\gamma$ a (product) Stolz path. If both $\gamma_{1}$ and $\gamma_{2}$ are tangential paths, then we term $\gamma$ a (product) tangential path. We define the principal cluster set of $f$ at $P=\left(e^{i \theta_{1}}, e^{i \theta_{2}}\right)$ as the intersection of all the cluster sets $C_{\gamma}(f, P)$, where the intersection is taken over all product paths $\gamma$ terminating at $P$.

Let $\Delta_{1}$ and $\Delta_{2}$ be two Stolz angles in $U$ which terminate at $e^{i \theta_{1}}$ and $e^{i \theta_{2}}$, respectively, and let $\Delta=\Delta_{1} \times \Delta_{2}$ denote the product Stolz angle which terminates at $P=\left(e^{i \theta_{1}}, e^{i \theta_{2}}\right)$. Then the cluster set $C_{\Delta}(f, P)$ is defined as the set of values $\zeta \in \mathbf{C}_{\infty}$ such that there is a sequence $\left\{\left(z_{n}, w_{n}\right)\right\}$ with the properties that $\left(z_{n}, w_{n}\right) \in \Delta$ for each $n,\left(z_{n}, w_{n}\right) \rightarrow\left(e^{i \theta_{1}}, e^{i \theta_{2}}\right)$, and $f\left(z_{n}, w_{n}\right) \rightarrow \zeta$. We define $C_{A}(f, P)$, the outer angular cluster set of $f$ at $P$, as the set

$$
C_{A}(f, p)=\bigcup C_{\Delta}(f, P),
$$

where the union is taken over all (product) Stolz angles $\Delta$ terminating at $\boldsymbol{P}$. A value $\zeta$ in $C_{A}(f, P)$ is called an (outer) angular cluster value of $f$ at $P$.

In the sequel, we also require the following definitions and terminology associated with certain one-sided approaches to a point $P$ on $T^{2}$. If $0<\eta \leqslant$ $\pi$, we call

$$
\Sigma_{s S}(\eta)=\{(z, w) \mid 3 \pi / 2-\arg (z-1) \leqslant \eta, 3 \pi / 2-\arg (w-1) \leqslant \eta\}
$$

the $\eta$-sector from the south-south. Henceforth, we tacitly assume without loss of generality that the point $P$ on $T^{2}$ under consideration is the point $(1,1)$. Let $\bar{C}_{S S}(\eta)$ denote the cluster set of $f \in H^{\infty}\left(U^{2}\right)$ at $(1,1)$ in the sector $\Sigma_{s S}(\eta)$, let

$$
C_{S S}(\eta)=\bigcup_{\eta^{\prime}<\eta} \bar{C}_{S S}\left(\eta^{\prime}\right)
$$

and let

$$
C_{s S}(0)=\bigcap_{\eta>0} C_{s S}(\eta)=\bigcap_{\eta>0} \bar{C}_{s S}(\eta) .
$$

Thus, $C_{S S}(0)$ is the set of tangential cluster values from the south-south of $f$ at $(1,1)$.

If $\eta>0$, we denote by $R_{s S}(\eta)$ the set of values assumed by $f$ arbitrarily near $(1,1)$ in some $\eta^{\prime}$-sector (from the south-south) with $\eta^{\prime}<\eta$, and we define $R_{S S}(0)=\bigcap_{\eta>0} R_{S S}(\eta)$. Finally, we define $\hat{B}_{S S}$, the distinguished boundary cluster set of $f$ at $(1,1)$ from the south-south, as the intersection 
$(n \geqslant 1)$ of the closure of the set of cluster values of $f$ at points $\left(e^{i \theta}, e^{i \phi}\right)$ on $T^{2}$ such that $-1 / n<\theta, \phi<0$. The sets $\Sigma_{S N}, \Sigma_{N N}$, etc., and the corresponding one-sided cluster sets, are defined analogously. (See [3] for a detailed discussion of some of the cluster sets defined above.)

3. The technique and the results with applications. We begin this section with some preliminary results of Doob [6] which, thanks to the one-sided approach, readily extend to the polydisc $U^{2}$.

Lemma 1. Let $f \in H^{\infty}\left(U^{2}\right)$. If $\alpha \in C_{S S}(0)$, then $|\alpha| \leqslant \sup |\beta|$, where the supremum is taken over all points $\beta$ in $\hat{B}_{S S}$.

THEOREM 2. Let $f \in H^{\infty}\left(U^{2}\right)$. Then the set $C_{S S}(0) \cap \tilde{\hat{B}}_{S S}$ is open.

Since the proofs of Lemma 1 and Theorem 2 involve only the iterations of certain one-variable ideas, we omit the proofs here. (For the details of the proofs in the one-variable case, we refer the reader to Doob [6].) We remark that mutatis mutandis analogous results hold for the other directions of approach. Thus, for example, Theorem 2 remains valid if $C_{s s}(0)$ is replaced by, say, $C_{S N}(0)$ and $\hat{B}_{S S}$ is replaced by the corresponding set $\hat{B}_{S N}$. Similar remarks apply to our subsequent results which we present for only one particular direction of approach. It is this property which is the raison d'être for our use of one-sided cluster sets.

The next result, Theorem 3, which we need in the sequel, is a generalization to the polydisc $U^{2}$ of Doob's extension of the Iversen Theorem [9]. This result contains the classical Iversen Theorem (see Doob [6, p. 463], and Collingwood and Lohwater [4, p. 89] and [2]). In addition to a standard normal family argument, the proof of Theorem 3 rests on a remarkable geometric property which is enjoyed by certain Möbius transformations. In the argument which follows, we briefly describe this property.

$\tilde{\hat{A}}$ We suppose, for the sake of argument, that there is a point $\alpha$ in $C_{S S}(\eta) \cap$ $\hat{B}_{s S}$ which is also a boundary point of $C_{s S}(\eta)$, where $\eta>0$. Then there is a sequence $\left\{\left(z_{n}, w_{n}\right)\right\}$ in the $\eta^{\prime}$-sector $\Sigma_{s s}\left(\eta^{\prime}\right)$, for some $\eta^{\prime}<\eta$, such that $\left(z_{n}, w_{n}\right) \rightarrow(1,1)$ and $f\left(z_{n}, w_{n}\right) \rightarrow \alpha$. Let $K_{n}$ and $L_{n}$ denote the Möbius transformations which map $U$ onto $U$ and satisfy the conditions $K_{n}(1)=L_{n}(1)=1$, $K_{n}(0)=z_{n}$, and $L_{n}(0)=w_{n}$. The properties of $K_{n}$ and $L_{n}$ now imply that there is a closed polydisc $\bar{D}$ about the origin such that for all $n$, the mappings $(z, w) \rightarrow\left(K_{n}(z), L_{n}(w)\right)$ take $\bar{D}$ into the interior of the sector $\Sigma_{s s}(\eta)$. Now we define $f_{n}(z, w)=f\left(K_{n}(z), L_{n}(w)\right)$, and we observe that since $f \in H^{\infty}\left(U^{2}\right)$, $\left\{f_{n}\right\}$ is a normal family. The rest of the argument is mutatis mutandis the same as in Doob [6, p. 467]. We obtain that $\alpha$ is in $C_{S S}(0)$. Since $C_{S S}(0) \cap \hat{B}_{S S}$ is open by Theorem 2, $\alpha$ cannot be a boundary point of $C_{s s}(\eta)$. This contradiction completes the proof of Theorem 3.

Theorem 3 (The Doob-Iversen Theorem). Let $f \in H^{\infty}\left(U^{2}\right)$. If $\eta>0$, then the set $C_{S S}(\eta) \cap \hat{\hat{B}}_{S S}$ is open. 
In the following key lemma, whose one-variable version is due to Doob [6, p. 463], we exhibit by a concrete construction the second ingredient of our technique. That is, in the proof of the key lemma we demonstrate the manner in which a sequence of functions in $H^{\infty}(U)$ can be constructed to reflect some of the desired properties of a single function in $H^{\infty}\left(U^{2}\right)$.

LemMa 4 (Key Lemma). Let $f \in H^{\infty}\left(U^{2}\right)$, and let $\alpha \in C_{S N}(0) \cap \tilde{\hat{B}}_{S N}$. Let $D\left(\alpha, \hat{B}_{S N}\right)$ denote the open component of $\mathbf{C}_{\infty}-\hat{B}_{S N}$ containing $\alpha$. Then, aside from at most one exceptional point, $R_{S N}(0) \supseteq D\left(\alpha, \hat{B}_{S N}\right)$.

Proof. By Theorem 2, the set $C_{S N}(0) \cap \tilde{\hat{B}}_{S N}$ is open. Hence, it follows that $D\left(\alpha, \hat{B}_{S N}\right) \subseteq C_{S N}(0)$. Let $\left\{\left(z_{n}, w_{n}\right)\right\}$ be a sequence of points in $U^{2}$ such that $\left\{\left(z_{n}, w_{n}\right)\right\}$ converges tangentially from the south-north to $(1,1)$, and such that $f\left(z_{n}, w_{n}\right) \rightarrow \alpha$ as $n \rightarrow \infty$. For each fixed $n$, we choose an open $\operatorname{arc} A_{n}=a_{n}$, of $T$ which abuts $z=1$ from the south, and we choose an open $\operatorname{arc} B_{n}=1, b_{n}$ of $T$ which abuts $w=1$ from the north. Moreover, we require that the lengths $\left\|A_{n}\right\|,\left\|B_{n}\right\|$ of the arcs $A_{n}$ and $B_{n}$, respectively, tend to zero, but so slowly that $\mu\left(z_{n}, A_{n}\right), \mu\left(w_{n}, B_{n}\right) \rightarrow 1$, where $\mu\left(z_{n}, A_{n}\right)$ denotes the harmonic measure of $A_{n}$ at $z_{n}$ with respect to the unit $\operatorname{disc} U$.

Next, for each $n$, we denote by $\phi_{n}$ the Möbius transformation which maps $U$ onto $U$ and satisfies the conditions $\phi_{n}\left(a_{n}\right)=1$ and $\phi_{n}\left(z_{n}\right)=w_{n}$. If $\left\|\phi_{n}\left(A_{n}\right)\right\|$ $\leqslant\left\|B_{n}\right\|$ for infinitely many $n$, then we set $f_{n}(z)=f\left(z, \phi_{n}(z)\right)$, and we consider the function-arc sequence $\left\{f_{n}, A_{n}\right\}$. (Otherwise, we let $c_{n}=\phi_{n}^{-1}\left(b_{n}\right)$, and we consider the function-arc sequence $\left\{g_{n}, B_{n}\right\}$, where $g_{n}(w)=f\left(\phi_{n}^{-1}(w), w\right)$.) We now proceed to show that this is the desired reduction to the one-variable situation.

Let $S$ denote the boundary cluster set of the sequence $\left\{f_{n}\right\}$ relative to the sequence of $\operatorname{arcs}\left\{A_{n}\right\}$. (For the definition of this cluster set, see Doob [6, p. 466].) Since $S \subseteq \hat{B}_{S N}$ and $\alpha \notin \hat{B}_{S N}$ by assumption, it is obvious that $\alpha \notin S$. Now the above construction implies that $f_{n}\left(z_{n}\right)=f\left(z_{n}, w_{n}\right) \rightarrow \alpha$ and $\mu\left(z_{n}, A_{n}\right)$ $\rightarrow 1$. Thus, it follows from a one-variable result of Doob (see [6, p. 466, Theorem 5.1] or [5, p. 441, Theorem 4.2]) that at most one point of $D(\alpha, S) \supseteq$ $D\left(\alpha, \hat{B}_{S N}\right)$ is omitted by the sequence $\left\{f_{n}\right\}$. Hence, aside from at most one point of $D\left(\alpha, \hat{B}_{S N}\right), f$ assumes every point of $D\left(\alpha, \hat{B}_{S N}\right)$. Finally, we observe that this conclusion remains valid if the domain of $f$ is replaced by the intersection of any neighborhood of $(1,1)$ with the interior of any $\eta$-sector $(\eta>0)$ from the south-north. This completes the proof of the key lemma.

LEMma 5. Let $f \in H^{\infty}\left(U^{2}\right)$. If $\alpha \in C_{s S}(\eta) \cap \tilde{\hat{B}}_{s S}, \eta>0$, and if $C_{s S}(0) \cap$ $D\left(\alpha, \hat{B}_{S S}\right)=\varnothing$, then

$$
\left[C_{s s}(\eta)-R_{s s}(\eta)\right] \cap D\left(\alpha, \tilde{\hat{B}}_{s s}\right)=\varnothing .
$$

Since the proof of Lemma 5 is similar to the proof of Theorem 3, we omit it.

We are now in a position to apply the above results and prove the Gross Cluster-Value Theorem for functions in $H^{\infty}\left(U^{2}\right)$. 
Theorem 6 (Gross Cluster-Value Theorem). Let $f \in H^{\infty}\left(U^{2}\right)$. Then every angular cluster value of $f$ at $(1,1)$ which is a limit point of $\tilde{R}(f,(1,1))$ is a principal value of $f$ at $(1,1)$.

Proof. Suppose the conclusion of the theorem is false. Then there is an angular sequence $\left\{\left(z_{n}, w_{n}\right)\right\}$ in $U^{2}$ with limit $(1,1)$ along which $f$ has limit $\alpha$, where $\alpha$ is a limit point of $\tilde{R}(f,(1,1))$, and there is a product path $\gamma=\gamma_{1} \times$ $\gamma_{2}$ terminating at $(1,1)$ such that $\alpha \notin C_{\gamma}(f,(1,1))$. Since in every locally compact, connected, and locally connected space any two points are the endpoints of a simple path in the space (see, for example, Whyburn [12, p. 36]), we may assume that $\gamma_{1}$ and $\gamma_{2}$ are simple. We further assume that $\gamma_{j}$ $(j=1,2)$ has its initial point, different from 1 , on $T$. Thus $\gamma$ divides $U^{2}$ into four product domains. By taking a suitable subsequence of the sequence $\left\{\left(z_{n}, w_{n}\right)\right\}$, if necessary, we may assume that the sequence lies in one of these domains, say $D=D_{1} \times D_{2}$. Finally, we assume without loss of generality that the portion of the boundary of $D_{j}$ which lies on $T$ abuts 1 from above. Now let $\phi_{j}$ map $D_{j}$ conformally onto $U$ such that $\phi_{j}(1)=1(j=1,2)$. Then it follows from a lemma of Doob [6, p. 464] that the sequence $\left\{\left(\phi_{1}\left(z_{n}\right), \phi_{2}\left(w_{n}\right)\right)\right\}$ lies in some $\eta^{\prime}$-sector from the south-south, where $\eta^{\prime}<\pi$. Thus, if we let $g(z, w)=f\left(\phi_{1}(z), \phi_{2}(w)\right),(z, w) \in D$, and choose $\eta>\eta^{\prime}$, then $\alpha \in C_{s S}(g, \eta)$ $\cap \hat{B}_{S S}(g)$ and $\alpha$ is a limit point of $\tilde{R}_{S S}(g, \eta)$.

In order to obtain a contradiction, we next examine two mutually exclusive cases. First, if $\alpha \in C_{S S}(g, 0) \cap \hat{\hat{B}}_{S S}(g)$, then by Lemma 4 the set $R_{S S}(g, 0)$, and $a$ fortiori the set $R_{S S}(g, \eta)_{2}$ contains at least a deleted neighborhood of $\alpha$. Second, if $\alpha \notin C_{S S}(g, 0) \cap \hat{B}_{S S}(g)$, then an easy argument shows that $C_{S S}(g, 0) \cap D\left(\alpha, \hat{B}_{S S}(g)\right)=\varnothing$. Hence, by Lemma $5, R_{S S}(g, \eta)$ contains at least a deleted neighborhood of $\alpha$. Since $\alpha$ was a limit point of $\tilde{R}_{S S}(g, \eta)$, we have arrived at a contradiction. Thus, the proof of the Gross Cluster-Value Theorem is complete.

Our first corollary is an immediate consequence of the Gross Cluster-Value Theorem.

Corollary 7. Let $f \in H^{\infty}\left(U^{2}\right)$. If $\sigma=\sigma_{1} \times \sigma_{2}$ is a product Stolz path in $U^{2}$ terminating at $(1,1)$, then

$$
C_{\sigma}(f,(1,1)) \cap \partial C(f,(1,1)) \subseteq C_{\gamma}(f,(1,1)),
$$

where $\gamma=\gamma_{1} \times \gamma_{2}$ is any product path in $U^{2}$ terminating at $(1,1)$, and where $\partial C(f,(1,1))$ denotes the boundary of the cluster set of $f$ at $(1,1)$. Moreover, if $C(f,(1,1))$ is nowhere dense, then the outer angular cluster set of $f$ at $(1,1)$ is equal to the principal cluster set of $f$ at $(1,1)$.

In order to underscore the scope of the foregoing results, we next present a slight extension of Lindelöf's classical theorem on the asymptotic values of analytic functions [10]. 
THEOREM 8. Let $f \in H^{\infty}\left(U^{2}\right)$, and suppose that $\|f\|_{\infty} \leqslant 1$. Let $\gamma=\gamma_{1} \times \gamma_{2}$ be a product path in $U^{2}$ terminating at $(1,1)$, and let $\Delta=\Delta_{1} \times \Delta_{2}$ be a product Stolz angle with vertex at $(1,1)$. Then there is a constant $\alpha=\alpha(\Delta), 0<\alpha<1$, such that

$$
\varlimsup_{\substack{(z, w) \rightarrow(1,1) \\(z, w) \in \Delta}}|f(z, w)| \leqslant\left[\varlimsup_{\substack{(z, w) \rightarrow(1,1) \\(z, w) \in \gamma}}|f(z, w)|\right]^{\alpha} .
$$

Given our earlier results, the proof of Theorem 8 involves only minor modifications of a technique of Cameron and Storvick [1], and so we omit the proof.

If in addition to the hypotheses of Theorem 8 we assume that the cluster set $C(f,(1,1))$ of $f$ at $(1,1)$ is nowhere dense, then we may take $\alpha$ to be 1 . This observation is a novelty, which we feel is worth mentioning even in the one-variable case. It is an immediate consequence of Corollary 7.

Corollary 9. Let $f \in H^{\infty}\left(U^{2}\right)$, and suppose that $\|f\|_{\infty} \leqslant 1$. If $C(f,(1,1))$ is nowhere dense, then $\varlimsup_{\lim _{\Delta}}|f(z, w)| \leqslant \varlimsup_{\overline{\lim }}|f(z, w)|$, where $\Delta$ and $\gamma$ have the same meaning as in Theorem 8.

We conclude this paper with an open question related to what we termed in the Introduction as the "Gehring-Lohwater phenomenon." In the one-variable case, Gehring and Lohwater [7] proved the following beautiful theorem.

Theorem 10 (The Gehring-Lohwater Theorem). Let $f=u+i v \in$ $H^{\infty}(U)$, and let $\alpha$ and $\beta$ be any two paths in $U$ terminating at 1 . If $u \rightarrow a$ and $v \rightarrow b$ as $z \rightarrow 1$ along $\alpha$ and $\beta$ respectively, then $f \rightarrow a+i b$ uniformly as $z \rightarrow 1$ in any Stolz angle.

The question is whether or not this generalization of Lindelöf's theorem remains valid in the polydisc $U^{2}$.

\section{BIBLIOGRAPHY}

1. R. H. Cameron and D. A. Storvick, A Lindelöf theorem and analytic continuation for functions of several variables, with an application to the Feynman integral, Proc. Sympos. Pure Math., vol. 11, Amer. Math. Soc., Providence, R. I., 1968, pp. 149-158.

2. C. L. Childress and G. L. Csordas, The Iversen theorem in a polydisc, Math. Scand. 42 (1978), 64-70.

3. The theory of cluster sets in the unit polydisc, Festschrift für Rolf Nevanlinna, Silivri (to appear).

4. E. F. Collingwood and A. J. Lohwater, The theory of cluster sets, Cambridge Tracts in Math. Physics, no. 56, Cambridge Univ. Press, Cambridge, 1966.

5. J. L. Doob, The boundary values of analytic functions, Trans. Amer. Math. Soc. 35 (1933), 418-451.

6. __ One-sided cluster-value theorems, Proc. London Math. Soc. (3) 13 (1963), 461-470.

7. F. W. Gehring and A. J. Lohwater, On the Lindelö theorem, Math. Nachr. 19 (1958), $165-170$.

8. W. Gross, Zum Verhalten der konformen Abbildung am Rande, Math. Z. 3 (1919), 43-64.

9. F. Iversen, Sur quelques propriétés des fonctions monogènes au voisinage d'un point singulier, 
Öfvers. Finska Vetensk. Soc. Forh. 58A, no. 25 (1915/16), 1-16.

10. E. Lindelöf, Sur un principe général de l'analyse et ses applications à la theorie de la représentation conforme, Acta Soc. Sci. Fenn. 46, no. 4 (1915), 1-35.

11. A. J. Lohwater, The boundary behaviour of analytic functions, Mathematical Analysis, Vol. 10, Akad. Nauk SSP Vsesojuz. Inst. Naučn. i Tehn. Informacii, Moscow, 1973. (Russian)

12. G. T. Whyburn, Analytic topology, Amer. Math. Soc. Collog. Publ., vol. 28, Amer. Math. Soc., Providence, R. I., 1967.

Department of Mathematics, Case Western Reserve University, ClevVeland, Ohio 44106

Department of Mathematics, Universtty of Hawall, Honolulu, HaWaII 96822 